\title{
A Case of Thyroid Hemiagenesis Associated With Graves' Disease and Follicular Neoplasm
}

\author{
Bu Kyung Kimª Ju Won Lee ${ }^{\mathrm{a}}$, Min Jung Jung ${ }^{\mathrm{b}}$, Eun Mi Lee ${ }^{\mathrm{a}}$, So Young Ock ${ }^{\mathrm{a}}$, \\ Su Kyoung Kwon ${ }^{\mathrm{a}}$, Young Sik Choi ${ }^{\mathrm{a}, \mathrm{c}}$
}

\begin{abstract}
Thyroid hemiagenesis is a rare congenital anomaly in which the thyroid lobe fails to develop, and the occurrence of thyroid hemiagenesis with other thyroid diseases is possible. Here, we report a case of thyroid hemiagenesis with Graves' disease and follicular neoplasm. Our patient, an 81-year-old female, was admitted with general weakness and weight loss over the previous month. Laboratory testing showed elevated free T4, suppressed TSH, and elevated TSH receptor antibody levels. A 99mTc thyroid scan showed absent uptake in the left lobe and a cold nodule on the isthmus of the thyroid gland. Ultrasound imaging showed an oval-shaped thyroid nodule on the isthmus and diffuse enlargement and hypervascularity of the right lobe. We performed ultrasound-guided fine needle aspiration, and cytologic analysis showed evidence of a Hurthle cell follicular neoplasm. To date, this is the first reported case of thyroid hemiagenesis associated with Graves' disease and Hurthle cell follicular neoplasm.
\end{abstract}

Keywords: Hemiagenesis; Graves' disease; Follicular neoplasm

\section{Introduction}

First reported by Handfield-Jones in 1866 [1], thyroid hemiagenesis is an uncommon congenital abnormality resulting from the developmental failure of a single thyroid lobe. Usually, the absence of one thyroid lobe does not result in clinical symptoms, so thyroid hemiagenesis is frequently diagnosed coincidently during the evaluation of other thyroid-related conditions. To date, approximately 330 cases have been reported, and half of them were associated with other disease in

Manuscript accepted for publication May 19, 2015

${ }^{\text {aDepartment }}$ of Internal Medicine, Kosin University College of Medicine, Busan, Korea

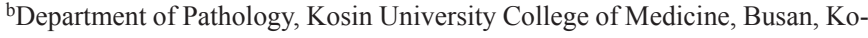
rea

${ }^{\mathrm{c} C}$ Corresponding Author: Young Sik Choi, Department of Internal Medicine, Kosin University College of Medicine, 34 Amnam dong, Seo-gu, Busan 602702, Korea. Email: yschoi@kosinmed.or.kr

doi: http://dx.doi.org/10.14740/jmc2189w the remnant thyroid lobe. However, few cases of hemiagenesis were comorbid for multiple diseases. Because we encountered a rare case of thyroid hemiagenesis associated with Graves' disease and a follicular adenoma, we present the case and literature review in this report.

\section{Case Report}

Our patient was an 81-year-old female who was admitted for fatigue and body weight loss for 1 month. Her past medical history was significant for Graves' disease diagnosed 6 years prior. After taking antithyroid medication for 1 year, she stopped and did not follow up until presenting with her current complaints. In the endocrinology clinic, she complained of general weakness, fatigue, and weight loss. Other significant medical history includes aortic stenosis (AS) and arterial fibrillation (AF). Also, surgical history includes left nephrectomy for kidney donation in 1993 and mitral valve replacement operation for rheumatic heart disease in 1993.

Initial vital signs were stable, with a blood pressure of $130 / 90 \mathrm{~mm} \mathrm{Hg}$, pulse of $62 / \mathrm{min}$, respiratory rate of $20 / \mathrm{min}$, and a body temperature of $36.5^{\circ} \mathrm{C}$. The thyroid gland was palpable with a firm and irregular margin.

Thyroid function testing revealed an FT4 level of $2.57 \mathrm{ng} /$ $\mathrm{dL}(0.89-1.76 \mathrm{ng} / \mathrm{dL}), \mathrm{T} 3$ level of $192.96 \mathrm{ng} / \mathrm{dL}(60-181 \mathrm{ng} /$ $\mathrm{dL})$, and a TSH level of $<0.008 \mu \mathrm{IU} / \mathrm{mL}(0.35-5.50 \mu \mathrm{IU} / \mathrm{mL})$. Antibody testing showed antithyroglobulin (anti-Tg), antithyroid peroxidase (anti-TPO), and TSH receptor antibody levels to be $500 \mathrm{IU} / \mathrm{mL}(0-4.9),>1,300 \mathrm{IU} / \mathrm{mL}(0-34)$, and $>405$ IU/L (0 - 10), respectively.

Ultrasound revealed a $1.7 \times 1.1 \mathrm{~cm}$ nodule in the right thyroid lobe, free of microcalcifications with regular margins. The thyroid parenchyma on the right side was heterogeneous and hyperechoic, but the tissue on the left side was not visible on ultrasound. Notably, the right lobe was connected to a visible isthmus, and there was a $1.5 \times 2.8 \mathrm{~cm}$ nodule with regular margins at the isthmus (Fig. 1). A Tc-99m pertechnetate thyroid scan further characterized the left thyroid hemiagenesis, diffuse right lobe enlargement, a cold nodule on the isthmus, and a warm nodule in the right lobe (Fig. 2). Fine needle aspiration biopsy of the large isthmus nodule was suspicious for a follicular neoplasm, specifically, Hurthle cell type, suspicious for Hashimoto's thyroiditis. The aspirate was cellular and con- 


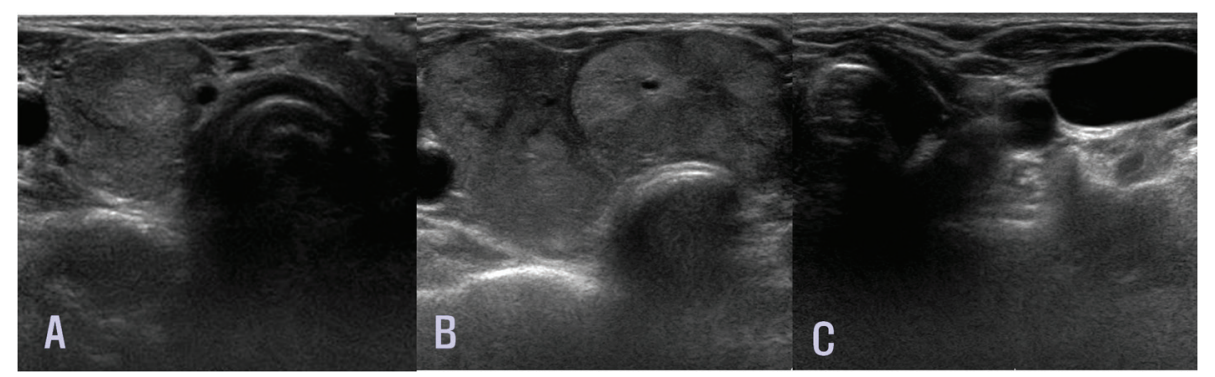

Figure 1. Ultrasound of the thyroid gland in the transverse plane. Large nodules are observed on the (A) right thyroid lobe and (B) isthmus. (C) Thyroid tissue on the left side is not visible.

sisted almost exclusively of loosely cohesive Hurthle cells. Their nuclei were enlarged, round, and central or eccentrically located with distinct nucleoli. Their cytoplasm was abundant, finely granular, and green or red in color on Papanicolaou stain. Colloid was absent, but a lymphocytic background was focally identified (Fig. 3).

After this testing, the patient was prescribed methimazole $10 \mathrm{mg}$ twice per day. After 4 days, the patient's symptoms were relieved. Thyroid function testing was performed again, and T3 level was $181.31 \mathrm{ng} / \mathrm{dL}(60-181 \mathrm{ng} / \mathrm{dL})$, TSH level was < $0.008 \mu \mathrm{IU} / \mathrm{mL}(0.35-5.50 \mu \mathrm{IU} / \mathrm{mL})$, and FT4 level was 1.54 $\mathrm{ng} / \mathrm{dL}(0.89-1.76 \mathrm{ng} / \mathrm{dL})$. Currently, the patient's progress is being observed on an outpatient basis, and the isthmus cold nodule is being managed conservatively with observation due to advanced age and medical history.

\section{Discussion}

Thyroid hemiagenesis is a rare congenital anomaly that was first reported in 1895 by Marshall [2]. The prevalence of thyroid hemiagenesis has been reported to be in the range of 0.05 $0.2 \%$ [3-6]. It occurs three times more often in women than men. Also, hemiagenesis occurs more frequently on the left thyroid lobe, almost $80 \%$ of the time, and is found on the isthmus about $50 \%$ of the time [7]. Consistently, this case of hemi- agenesis was also found on the left thyroid lobe of a woman.

Embryologically, the thyroid gland is the first endocrine organ to develop, and it can be observed as early as the third week of gestation. The gland originates from the proliferation of endodermal epithelium on the median surface of the developing pharyngeal floor. It is derived from the ventral endodermal diverticulum from the first and second pharyngeal pouches and is located midline in the floor of the pharynx or in the foramen cecum. The diverticulum descends from the base of the tongue to the adult pre-tracheal position via a midline anterior path during weeks 4 - 7 of gestation [8].

The cause of thyroid hemiagenesis is unknown, although there are several possible explanations. Hemiagenesis could be a result of an impairment of descent or due to a defect of lobulation. Because there is no compensatory growth of the remaining lobe, a lobulation defect rather than impairment of descent appears to the more likely mechanism [9]. Notably, the occurrence of hemiagenesis in monozygotic twins and members of the same family suggests a genetic component [10]. A small but significant proportion of familial thyroid dysgenesis cases have been identified through the study of subjects with congenital hypothyroidism, and familial thyroid dysgenesis was noted in $40 \%$ of one series of patients with hemiagenesis [11]. These findings support a possible role for genetic factors in thyroid development.

Based on established reports, the incidence of comorbid

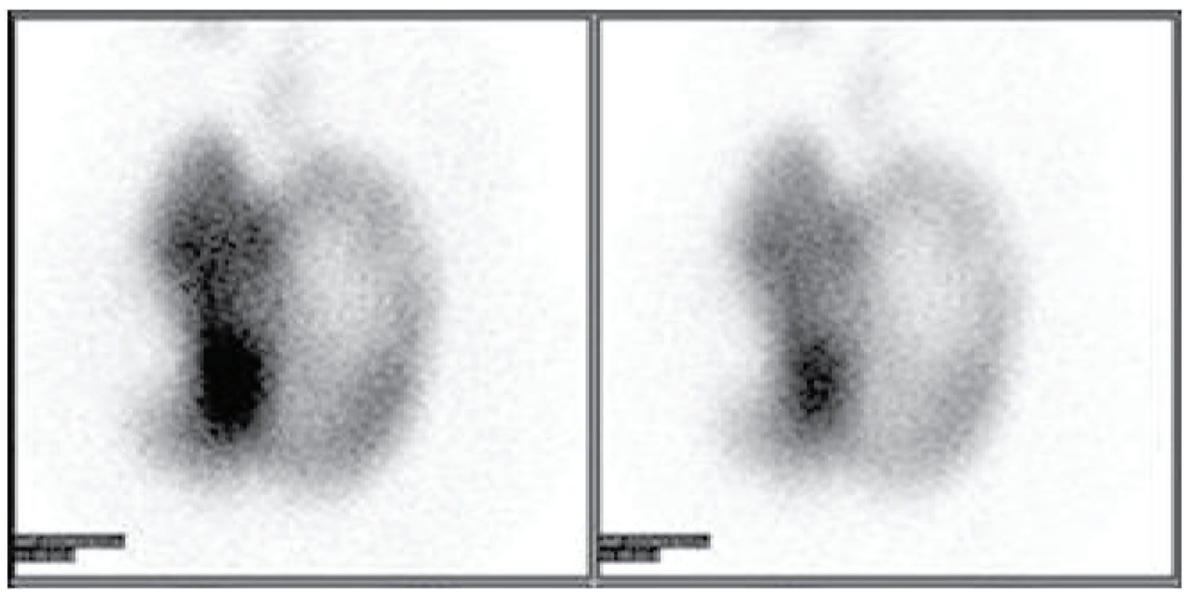

Figure 2. A radionuclide thyroid scan with $99 \mathrm{mTc}$ demonstrated left thyroid hemiagenesis, diffuse right lobe enlargement, a cold nodule on the isthmus, and a right-sided warm nodule. 


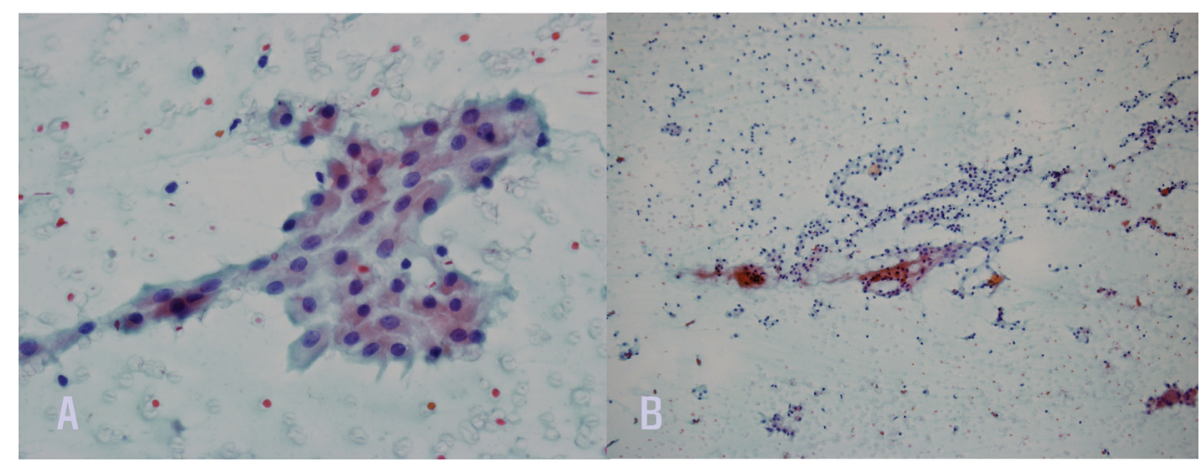

Figure 3. Cytologic findings. (A) Hurthle cells exhibit enlarged, round, and central or eccentrically located nuclei with distinct nucleoli and abundant, finely granular, green or red cytoplasm ( $\times 400$, Papanicolaou stain). (B) The aspirate showed Hurthle cells arranged in loose clusters. Colloid was absent, and a lymphocytic background was focally identified ( $\times 100$, Papanicolaou stain).

thyroid disease in patients with thyroid hemiagenesis is greater than 50\%. Among these comorbid diseases, hyperthyroidism occurred in $8.5 \%$ of cases, and multinodular goiter was found in $2.7 \%$. However Graves' disease and multinodular goiter were reported in only one case [12].

This case is the first case of thyroid hemiagenesis associated with Graves' disease and follicular neoplasm. The patient had multinodular goiter and a cold nodule on the isthmus. The follicular neoplasm was diagnosed cytologically by fine needle aspiration. Unfortunately, we did not pathologically confirm the type of carcinoma or adenoma because of her comorbid diseases, AS and AF, and her medication profile.

Thyroid hemiagenesis is an uncommon congenital anomaly. Because hemiagenesis is asymptomatic, it is detected coincidently during imaging for other thyroid diseases. Although thyroid hemiagenesis is a benign condition, being unaware of its presence may lead to incorrect assumptions during clinical decision-making. Thus, it is necessary to report, collect, and establish data about thyroid hemiagenesis.

\section{References}

1. Peteiro-Gonzalez D, Cabezas-Agricola JM, Casanueva FF. [Thyroid hemiagenesis: report of five cases and literature review]. Endocrinol Nutr. 2013;60(8):e15-17.

2. Marshall CF. Variations in the Form of the Thyroid Gland in Man. J Anat Physiol. 1895;29(Pt 2):234-239.

3. Mikosch P, Gallowitsch HJ, Kresnik E, Molnar M, Gomez I, Lind P. Thyroid hemiagenesis in an endemic goiter area diagnosed by ultrasonography: report of sixteen patients. Thyroid. 1999;9(11):1075-1084.

4. Shabana W, Delange F, Freson M, Osteaux M, De Schepper J. Prevalence of thyroid hemiagenesis: ul- trasound screening in normal children. Eur $\mathrm{J}$ Pediatr. 2000;159(6):456-458.

5. Maiorana R, Carta A, Floriddia G, Leonardi D, Buscema M, Sava L, Calaciura F, et al. Thyroid hemiagenesis: prevalence in normal children and effect on thyroid function. J Clin Endocrinol Metab. 2003;88(4):1534-1536.

6. Gursoy A, Anil C, Unal AD, Demirer AN, Tutuncu NB, Erdogan MF. Clinical and epidemiological characteristics of thyroid hemiagenesis: ultrasound screening in patients with thyroid disease and normal population. Endocrine. 2008;33(3):338-341.

7. Melnick JC, Stemkowski PE. Thyroid hemiagenesis (hockey stick sign): a review of the world literature and a report of four cases. J Clin Endocrinol Metab. 1981;52(2):247-251.

8. Fagman H, Nilsson M. Morphogenesis of the thyroid gland. Mol Cell Endocrinol. 2010;323(1):35-54.

9. Karabay N, Comlekci A, Canda MS, Bayraktar F, Degirmenci B. Thyroid hemiagenesis with multinodular goiter: a case report and review of the literature. Endocr J. 2003;50(4):409-413.

10. Clifton-Bligh RJ, Wentworth JM, Heinz P, Crisp MS, John R, Lazarus JH, Ludgate M, et al. Mutation of the gene encoding human TTF-2 associated with thyroid agenesis, cleft palate and choanal atresia. Nat Genet. 1998;19(4):399-401.

11. Castanet M, Leenhardt L, Leger J, Simon-Carre A, Lyonnet S, Pelet A, Czernichow P, et al. Thyroid hemiagenesis is a rare variant of thyroid dysgenesis with a familial component but without Pax 8 mutations in a cohort of 22 cases. Pediatr Res. 2005;57(6):908-913.

12. Wu YH, Wein RO, Carter B. Thyroid hemiagenesis: a case series and review of the literature. Am J Otolaryngol. 2012;33(3):299-302. 